\title{
Research Article \\ Number of People who Inject Drugs in Son La, Vietnam: Population Size Estimation Based on Official Records
} \author{
Abu S. Abdul-Quader, ${ }^{* *}$ \\ ${ }^{1}$ Department of HIV/AIDS, National Institute of Hygiene and Epidemiology, Hanoi, Vietnam \\ ${ }^{2}$ Division of Global HIV and TB, US Centers for Disease Control and Prevention, Hanoi, Vietnam \\ ${ }^{3}$ Program Development Office, United States Agency for International Development, Hanoi, Vietnam \\ ${ }^{4}$ Division of Global HIV and TB, US Centers for Disease Control and Prevention, Atlanta, GA 30329, USA
}

Le Anh Tuan ${ }^{1}$, Nguyen Thi Thanh $\mathrm{Ha}^{1}$, Quan Dinh Nguyen², Giang Tong Le ${ }^{3}$, Duong Cong Thanh ${ }^{2}$, Meade Morgan ${ }^{4}$,

\section{ARTICLE INFO}

\section{Article History}

Received 26 March 2020

Accepted 05 May 2020

Keywords

Population size estimation people who inject drugs Vietnam

\begin{abstract}
Aims: Estimated population sizes of key populations are critical for resource allocation as well as for monitoring program performance to prevent HIV infection. In 2018, using official records we recruited and conducted a brief survey among People Who Use Drugs (PWUD) to estimate the population size of People Who Inject Drugs (PWID) in Son La Province, Vietnam.

Methods: In Son La, the authorities develop and maintain a master list of individuals who are suspected of using drugs. The list is updated quarterly and is used to monitor the drug use situation in the province. The list, however, does not distinguish injecting from non-injecting drug users. Individual lists from six districts, 24 communes, and 96 hamlets were selected from among those from 12 districts, 204 communes, and 3335 hamlets in the whole province. After reviewing the lists with the hamlet health workers, based on the length of the lists, a minimum of $20 \%$ of the total or five drug users in any hamlet with fewer than 25 PWUD were randomly selected for a brief assessment. In addition to basic demographics, the assessment included any drug use in the last 3 months, injection drug use in the last 3 months, and the last time injected.

Results: A total of 250 PWUD were interviewed by hamlet health workers. The total number of PWID across all 12 districts in Son La was estimated at 4475 [95\% Confidence Interval (CI): 3379-5570] and the corresponding proportion of PWID among PWUD was $48.4 \%$ (95\% CI: 36.6-60.4).

Conclusion: The exercise provided an estimated number of PWID in Son La. For planning HIV prevention and care service needs among PWID, additional studies using different methods are needed to validate and improve the population size of PWID in the province of Son La.
\end{abstract}

(C) 2020 The Authors. Published by Atlantis Press International B.V.

This is an open access article distributed under the CC BY-NC 4.0 license (http://creativecommons.org/licenses/by-nc/4.0/)

\section{INTRODUCTION}

The HIV epidemic in Vietnam is concentrated among Key Populations (KP) including People Who Inject Drugs (PWID), Men who have Sex with Men (MSM), female sex workers and their clients, and sex partners of people living with HIV [1]. Population Size Estimates (PSE) of KPs in Vietnam provide much-needed data to help policymakers and program administrators understand the scope of the HIV epidemic, help plan appropriate interventions, and allocate sufficient resources. PSE are used as denominators in calculating program coverage and producing national estimates and projections of the HIV epidemic in Vietnam. PSE data are needed to help the national- and provincial-level authorities and other stakeholders with disease control activities, specifically for program planning and assessments.

To meet the data needs, a PSE study of PWID was conducted in Son La Province, Vietnam. Son La is a mountainous province in

Corresponding author.Email: afa3@cdc.gov

Data availability statement: The data that support the findings of this study are available from the corresponding author $[\mathrm{AAQ}]$, upon reasonable request. the North-West part of the country. There are 12 districts and a total of 204 communes in Son La. HIV epidemic in Son La is driven primarily by injection drug use. In 2017, HIV prevalence among PWID in Son La was estimated to be 21\% [1]. According to the Son La authorities, there are 9238 PWUD in the province [1]. This number is based on the records developed by the law enforcement authorities, using data collected by the hamlet and commune leaders.

Many provinces in Vietnam have set up steering committees that are responsible for the prevention and control of crime, drug use, sex work, and HIV/AIDS. The committees operate at various levels (provincial, district, and commune) and are usually led by the deputy president of the people's committee, and includes members of the people's committee, police, health centers, and women's and youth union. The committees maintain lists of drug users with names, dates of birth, and addresses for each hamlet. The hamlet leaders are instrumental in generating this list at the hamlet level. The hamlet lists are then combined to generate the commune list which is then used to create the district and a master provincial list. The lists are expected to be 
updated when someone dies or goes to jail or moves permanently to another province or district. These data, however, do not always reflect the actual population size of PWID as the indicators used by the leaders can be biased and they do not separate those who inject from those who do not. They provide the estimated number of PWUD in the province irrespective of the mode of use.

While the lists generated by the hamlet leaders do not distinguish between injecting and non-injecting drug users, they are also subject to multiple biases. The lists are based on the knowledge and perception of the hamlet leaders. No sample surveys are conducted with hamlet residents to assess drug use and estimate the number of drug users in the hamlets.

Lists of drug users are available for all levels in Son La province and program planners had to rely on these lists to plan and develop programmatic activities. The lists in Son La include people who inject and who do not inject. More reliable and valid estimates are needed to appropriately plan and develop responses to the epidemic. In this paper, we describe a process, based on the available hamlet lists, to estimate the population size of PWID in Son La. The study was conducted in 2018.

\section{MATERIALS AND METHODS}

Compared with other urban areas such as in Hanoi and Ho Chi Minh City, PWUD in Son La are not always visible and accessible. In addition to the provincial topography, which makes it difficult to reach them, there is a strong presence and influence of law enforcement authorities at the district as well as at the commune levels. Villages and communes are praised and highlighted for becoming and maintaining drug-free statuses. These make it difficult to use any of the established PSE methods such as capture-recapture or survey or service multiplier. Capture-recapture requires access to the study population [2]. Surveys based on the Respondent-driven Sampling (RDS) method can provide data to calculate the estimated population size [3]. However, RDS surveys require setting up recruitment and interviewing sites, and the participants being able to have free access to the study sites for inclusion in the study [4]. A combination of both topographical settings and the law enforcement situation led to the use of existing hamlet lists and use that to reach PWUD to estimate the population size.

\subsection{Study Design}

Study populations included men and women aged 18 years or above who reported injecting illicit drugs (not prescribed for treatment purposes) within the previous 90 days and who had lived in the province for the past 6 months. Since the official lists do not reflect the actual estimated number of PWID in the province, the following process was used to estimate the population size of PWID in Son La.

\subsubsection{Selection of districts}

There are 12 districts in the province. Based on the reported numbers of PWUD from Son La Provincial Department of Public
Security, the districts were ordered from highest to lowest then grouped into three groups: high, medium, and low. A total of six districts-two from high, two from the medium, and two from low-were randomly selected. The number of PWUD in the six selected districts accounted for $55 \%$ of the total number of PWUD in the province.

\subsubsection{Selection of communes and hamlets}

After selection of the districts, all communes within each district were listed and four communes from each of the six districts were randomly selected with equal probability. Once the communes were selected, all hamlets within each commune were listed and four hamlets from each of the selected communes were randomly selected with equal probability. A total of six districts, 24 communes, and 96 hamlets were selected from among 12 districts, 204 communes, and 3335 hamlets in whole provinces.

\subsubsection{Drug user selection and assessment}

Once the hamlets were selected, the lists of PWUD developed by the hamlet leaders were reviewed by the study team along with the hamlet health workers. Based on the length of the list, a minimum of $20 \%$ of the total or five PWUD in any hamlets with fewer than 25 PWUD on the list were randomly selected for a brief assessment.

Once selected, hamlet health workers reached out to the selected person and conducted the assessment. The assessment included the collection of the following information: gender, age, hamletresidence or not, years residence of the hamlet, any drug use in the last 3 months, injection drug use in the last 3 months, last injected, and tested for HIV in last 6 months.

\subsection{Sample Size}

The target sample size was based on conservatively estimating the proportion of drug users who inject as $50 \%$ with a precision of $\pm 10 \%$, allowing for a survey design effect of 2.5 . A total of 250 drug users in 96 hamlets were interviewed by the hamlet health workers.

\subsection{Statistics Analysis}

Estimates of the proportion of PWID for each of the six sample districts were calculated among those reporting drug use in the past 3 months using sample survey methods which accounted for the twostage cluster design (communes and hamlets), with a finite population correction accounting for the number of communes. These were applied to the reported number of drug users (injecting and non-injecting, whether in the past 3 months or longer) to estimate the number of PWID in the districts. For the six non-sampled districts, an overall estimate of the proportion of PWID was calculated based on the pooled data from the sampled districts accounting for the three-stage design (district, commune, and hamlet), but without a finite population correction. This estimate was applied to the 
Table 1 Estimates of PWID based on hamlet list and brief assessments

\begin{tabular}{|c|c|c|c|c|c|c|}
\hline Districts & Communes & Hamlets & $\begin{array}{l}\text { Number of } \\
\text { reported } \\
\text { PWUD }^{*}\end{array}$ & $\begin{array}{c}\text { Average number } \\
\text { of PWUD } \\
\text { per Hamlet }\end{array}$ & $\begin{array}{l}\text { Percentage of } \\
\text { PWUD who } \\
\text { inject (PWID) }\end{array}$ & $\begin{array}{l}\text { PWID population } \\
\text { size, } n(95 \% \mathrm{CI})\end{array}$ \\
\hline Moc Chau & 15 & 213 & 2033 & 9.5 & $33.3(>0.0,70.0)$ & $678(>0,1423)$ \\
\hline Thuan Chau & 29 & 568 & 1315 & 2.3 & $79.2(56.5,100.0)$ & $1041(743,1315)$ \\
\hline Van Ho & 14 & 156 & 811 & 5.2 & $10.3(>0.0,20.8)$ & $83(>0,169)$ \\
\hline Sop Cop & 8 & 128 & 422 & 3.3 & $42.9(32.2,53.5)$ & $181(136,226)$ \\
\hline Song Ma & 19 & 466 & 1256 & 2.7 & $71.4(57.9,85.0)$ & $897(727,1067)$ \\
\hline Yen Chau & 15 & 217 & 400 & 1.8 & $35.3(15.2,55.4)$ & $141(61,222)$ \\
\hline Other - non sampled (six total) & 104 & 1587 & 3001 & 1.9 & $48.4(24.6,72.2)$ & $1454(738,2167)$ \\
\hline Total & 204 & 3335 & 9238 & 2.8 & $48.4(36.6,60.4)$ & $4475(3379,5570)$ \\
\hline
\end{tabular}

*Reported and included in the list of drug users by the hamlet leaders.

number of reported drug users in the six non-sampled districts. The total number of PWID was then calculated as the sum across the sampled and non-sampled districts, with a Confidence Interval (CI) based on the sum of the individual variances. All analyses were done in SAS version 9.4 and Excel.

\subsection{Human Subject Approval}

The study protocol received ethical approval from the Institutional Review Board of the National Institute of Hygiene and Epidemiology, Hanoi, Vietnam. The protocol was also reviewed in accordance with the US Centers for Disease Control and Prevention (CDC) human research protection procedures and was determined research, but CDC investigators did not interact with human subjects nor have access to identifiable data or specimens for research purposes.

\section{RESULTS}

A total of 250 PWUD from the hamlet lists were selected and interviewed by the hamlet health workers. An overwhelming majority (96\%) of the PWUD were men. The mean age was 40 years. About $40 \%$ of those interviewed mentioned having received an HIV test in the past 12 months. The number of reported PWUD in the selected districts varied widely from 400 in Yen Chau district to 2033 in Moc Chau district. Similar variations were found in terms of the proportion of PWUD who inject as well as the estimates of the number of PWID. The estimated proportion of PWUD who inject ranged from $10.3 \%$ in Van Ho district to $79.2 \%$ in Thuan Chau district while the estimated number of PWID ranged from 83 in Van Ho district to 1041 in Thuan Chau district (Table 1).

The proportion of PWUD who inject in the six non-sampled districts was estimated as $48.4 \%$ giving an estimated number of PWID of 1454 . The estimated number of PWID in the six sampled districts (3021) was substantially more than those in the non-sampled districts due in part to the smaller number of registered drug users (6237 vs. 3001). The total number of PWID across all 12 districts in Son La was estimated at 4475 (95\% CI: 3379-5570) and the corresponding proportion of PWID among PWUD was 48.4\% (95\% CI: 36.6-60.4).

\section{DISCUSSION AND LIMITATIONS}

This was the first study estimating the population size of PWID in Son La Province, Vietnam. Previous estimates of numbers of drug users were based on official records collected by hamlet leaders and those estimates did not distinguish injecting from non-injecting drug users. Additionally, the available official records were not based on any objective criteria or randomly selected survey of the hamlet residents. Rather, the hamlet leaders used their knowledge and understanding of drug use and who should be included in the list as drug users.

The estimated PWID population size relies on the accuracy of the counts of drug users provided by the hamlet and commune heads which was not formally assessed. As the data on lifetime drug use were not collected, the estimates of the proportion of injecting drug users from among all drug users over the past 3 months may be an inaccurate estimate of the proportion of injectors over their lifetime of drug use. The reported number of drug users in the province is much higher than the estimated number of those who are injecting drug users. As this study is based on the available list of drug users, additional studies, using multiple methods, are needed to arrive at a more robust estimate of PWID in the province. Because of the topography of the province and geographic isolation of some of the remote areas, PWID are not always accessible to service providers. Issues related to law enforcement also makes it difficult for those who inject to access services. Since Son La has a high prevalence of HIV among PWID it would be important to use non-formal avenues and local community-based organizations to reach and provide health services.

The hamlet leaders are encouraged to use their perceived knowledge to develop the lists.

Even with the limitations of the estimation, the study highlights the utility of existing official records. It also highlights the feasibility of using this method in other provinces in Vietnam where the implementation of available PSE methods has limitations due to law enforcement issues and/or nature of the drug use. Questions can also be raised whether official records can be sufficient when no estimates are available, or PSE cannot be implemented. 


\section{CONFLICTS OF INTEREST}

The authors declare they have no conflicts of interest.

\section{AUTHORS' CONTRIBUTION}

AAQ, LAT and GTL conceptualised the study. LAT and QDN conducted field work and collected the data. QDN, MM and AAQ analysed the data. AAQ and MM conducted additional analyses and drafted the manuscript. DCT, LAT, GTL and NTTH reviewed the findings and contributed to the draft of the manuscript. All authors reviewed, made comments and contributed to the overall study design, implementation, and draft of the manuscript. AAQ and MM edited and finalised the draft.

\section{FUNDING}

This study has been supported by the President's Emergency Plan for AIDS Relief (PEPFAR) through the U.S. Centers for Disease Control and Prevention (CDC) under the terms of 5U2GGH001628-02.

\section{DISCLAIMER}

The findings and conclusions in this report are those of the authors and do not necessarily represent the official position of the funding agencies.

\section{REFERENCES}

[1] Vietnam Authority of HIV/AIDS Control. Department of HIV/ AIDS Prevention and Control. Hanoi, Vietnam: VAAC, Ministry of Health; 2018.

[2] WHO, CDC, UNAIDS, FHI 360. Biobehavioral survey guidelines for populations at risk for HIV. Geneva: World Health Organization; 2017.

[3] Handcock MS, Gile KJ, Mar CM. Estimating hidden population size using respondent-driven sampling data. Electron J Stat 2014;8;1491-521.

[4] Heckathorn DD. Respondent-driven sampling II: deriving valid population estimates from chain-referral samples of hidden populations. Soc Probl 2002;49;11-34. 